\title{
Comunicación

\section{Consumo y usos de la televisión en los mercados públicos de la ciudad de México}

Este trabajo presenta los resultados de un primer acercamiento al consumo y a los usos de la televisión en los mercados públicos de la ciudad de México. ${ }^{2}$ Cabe destacar que en las 16 delegaciones en que se subdivide administrativamente la ciudad se distribuyen 317 mercados. La investigación se desarrolló en dos etapas: primero indagando cuantitativamente $10 \%$ de los mercados de cada delegación, con el fin de constatar y cuantificar la presencia de aparatos de televisión y el tipo de consumo que se estaba desarrollando en el momento del estudio, $\mathrm{y}$ segundo, realizando una observación participante para documentar los tipos de usos sociales de la televisión en los mercados públicos.

PALABRAS CLAVE: televisión, audiencias, consumo cultural, usos sociales, espacio público, mercados públicos.
This paper analyzes the market of the pay television industry in Mexico. The main objective of this research is, firstly, to generate statistical information to measure the economic and market concentration of the sub-sector in the face of changes that are being established with the technological convergence and triple play (voice, video and Internet in one network and transmission infrastructure). It also presents a brief historical review of the subsector to understand more fully the particularities of the Mexican case.

KEY WORDS: television, audiences, consumption, social uses, public space, public market.

1 Universidad Autónoma de la Ciudad de México.

Correo electrónico: jerorepoll@yahoo.com

2 Una versión preliminar de este artículo se presentó como ponencia en el Grupo de Trabajo de Estudios de Recepción de la Asociación Latinoamericana de Investigadores de la Comunicación (ALAIC). México, 2008. 
La presencia constante y hasta impertinente de los mensajes provenientes de emisores difusos, pero concretados en imágenes, señalamientos, indicaciones y símbolos constituyen el nuevo y cada vez más totalizante equipamiento urbano, cuyo rasgo más prominente es el avasallamiento de los sentidos, las visiones, las escuchas, las sensaciones (Orozco, 1997, p. 48).

\section{INTRODUCCIÓN}

Una revisión exhaustiva de la producción de estudios de recepción nos permitió concluir que, en este subcampo, se trabaja con el supuesto básico de que la interacción con la televisión se desarrolla en el contexto del hogar. Por otro lado, las interacciones individuales, a partir de la proliferación de aparatos de televisión en el mismo hogar y de la recepción de señal en otros soportes (PC, celulares), señalan que la familia ya no puede considerarse la unidad mínima de análisis. Limitar la interacción con la televisión al ámbito privado del hogar y en familia implica desconocer, al menos en la ciudad de México, otro abanico de interacciones que se da en el espacio público.

Si bien, como señalan Grimson, Masotta y Varela (1999) "la televisión se ha urbanizado naturalizando su incorporación en los espacios públicos de la ciudad" (p. 197). De las 28 entradas sobre estudios de recepción en la sistematización documental 1995-2001 de la investigación académica de la comunicación en México (Fuentes, 2003) ninguna hace referencia a la interacción entre audiencias y televisión en espacios públicos. El mismo resultado se obtiene tras revisar las 109 entradas referentes a estudios sobre televisión de la sistematización documental 1956-1986 (Fuentes, 1988). Finalmente, los 41 registros sobre estudios de recepción catalogados en la base de datos del Consejo Nacional para la Educación y la Investigación de las Ciencias de la Comunicación (CONEICC) de 2008 tampoco arrojan ningún resultado sobre estudios de recepción en espacios públicos. 
Por su parte, Guillermo Orozco y Rebeca Padilla, en el artículo Los estudios de recepción en México. Un itinerario (2005) señalan como grandes líneas de investigación de la recepción en México, la denuncia de los contenidos y sus posibles efectos, recepción y educación; la recepción y la construcción del espacio público; la recepción televisiva en familia y la recepción ante la programación propia y ajena. En este balance tampoco hacen referencia alguna a los estudios de recepción en espacios públicos, lo cual refrendan Orozco y González (2009), al no identificar ningún estudio de recepción en espacios públicos en la investigación documental que sustenta el texto Cuatro décadas de analizar la recepción de medios en México.

Esto se complementa, por otro lado, con los resultados a los que llegan Lozano y Frankenberg (2008) en su estudio sobre Los enfoques teóricos y estrategias metodológicas en la investigación empírica de audiencias televisivas en América Latina: 1992-2007, cuando señalan que:

La investigación empírica de audiencias en América Latina sigue siendo escasa (...y) la tendencia de los académicos latinoamericanos a optar por ensayos teóricos en lugar de trabajo empírico, ya sea por falta de recursos económicos o deficiente capacitación metodológica, ha permanecido presente en los últimos 15 años (pp. 100-101).

No obstante este panorama, queremos destacar la reciente aparición del trabajo de Sandy Rodríguez (2009) sobre el consumo de televisión en tres espacios públicos del Distrito Federal en el marco de su tesis de doctorado. Esta tesis, junto con la investigación que nutre este trabajo, se inscribe en el marco de estudios del gusto cultural, específicamente de la televisión. Consumo cultural que se diferencia del consumo centrado en el valor de cambio en la apropiación y usos de productos con estructuras de significado. Es decir, así como la industria cultural se diferencia de cualquier otra por el carácter de sus productos, el empleo de estos productos es necesariamente diferente de otro tipo de mercancías.

En el ámbito internacional también encontramos pocas experiencias. Hasta el momento hemos podido ubicar el trabajo de Anna McCarthy (2001), quien en Estados Unidos analiza cómo la televisión regula el flujo y la experiencia del tiempo en zonas públicas de tránsito, trabajo 
y servicio. Rodríguez (2009), subraya del trabajo de McCarthy que "la pantalla hace de la experiencia de esperar una actividad, pero además designa un espacio de espera. Es así como el ver televisión es una forma legitimizada para pasar el tiempo" (p. 8).

Mientras tanto, en América Latina, hallamos dos trabajos. El primero, de Grimson et al., (1999), describe la naturalización de la televisión en los espacios públicos de Buenos Aires y, en un segundo momento, analiza los usos de la televisión en trenes y subterráneos (metro) diferenciándolos de los usos de la televisión en bares. Con mínimas variantes, los usos en torno al transporte público coinciden con los indicados por McCarthy. Los usos de la televisión en los bares responden a las distintas características de éstos y sus respectivos clientes. En el artículo, en particular, se analiza un bar de "tránsito", donde "el grado de familiaridad propio del consumo televisivo privado se expande en el ámbito público del bar" (p. 215) y un bar de:

"Encuentro y prácticas lúdicas", donde "uno se siente como en un club ... perteneciente a una cultura futbolística masculina. Se realiza así una apropiación en público de la televisión, y esta apropiación tiene su modo particular impuesto por los socios del club" (pp. 221-222).

El segundo estudio, de Gastaldo, Leistner, Da Silva y McGinity (2006), se centra en el bar como lugar de observación, describiendo el comportamiento de los aficionados de fútbol durante la recepción de estos partidos en bares de Brasil: la distribución de las mesas en torno al televisor, las reacciones frente a los acontecimientos del partido y los comentarios respectivos. Estos apuntes, al tratarse de una investigación en curso, los autores señalan que sus resultados son preliminares y deben leerse en esos términos. No obstante, señalan que, "además del fuerte recorte de género, compartir un mismo juego estimula la formación de un espacio de socialización muy peculiar" (p. 168), lo cual coincide con la descripción de los "bares de encuentro" descritos en el artículo de Grimson et al.

Por otro lado, a través de la investigación documental que sustenta el libro Arqueología de los estudios culturales de audiencia (Repoll, 2010), he podido constatar el desplazamiento del texto al contexto, haciéndose cada vez más evidente la necesidad de analizar no sólo la 
lectura que de los textos hace la audiencia sino también el contexto en el que éstas interactúan con los mismos. Y es en esta preocupación por el contexto que se superó la perspectiva monocausal (Morley, 1996), que señalaba a la clase social como la dimensión determinante en la percepción-lectura de las audiencias, a favor de una mirada compleja, en torno a las prácticas sociales de la vida cotidiana en donde la televidencia ${ }^{3}$ tiene lugar, entretejida con las múltiples actividades, relaciones y deseos de las audiencias. Pero -y he aquí otra conclusión del mismo trabajo-, la familia ya no es la única ni mucho menos la exclusiva unidad mínima de análisis $\mathrm{y}$, por otro lado, tampoco es el hogar puertas adentro el lugar propio de la interacción de las audiencias con la televisión. En este sentido, como ya lo ha señalado Tufte (1997), la cotidianidad latinoamericana denuncia los límites de las propuestas analíticas que, como las de Silverstone (1996) conciben a la televisión y su consumo como una práctica doméstica, propia del espacio privado, típica de los suburbios de las grandes metrópolis. Esto, desde que llegué a la ciudad de México, me resultó -como al propio Tufte respecto de su experiencia en Asunción del Paraguay-, una descripción discordante con lo que se podía ver en las calles. La propuesta de Silverstone, entonces, aunque sugerente en muchos aspectos, se demuestra limitada y limitante para pensar las relaciones entre televisión y audiencias en Latinoamérica.

Si bien constatamos en la ciudad de México un repliegue sobre lo privado gracias a la creciente inseguridad del ciudadano en el espacio público y, en consecuencia, la consolidación de los medios como el espacio público desde donde se ve y comparte la ciudad (Repoll, 2008), esto no nos debe llevar a concluir que ésta es la única práctica de interacción de los capitalinos con la televisión. Junto a la inseguridad avasallante, el tráfico y los largos desplazamientos constituyen una de las características más destacadas de una ciudad y su área metropolitana, que supera los 20 millones de habitantes, donde éstos invierten un promedio de dos horas diarias en el traslado de su casa al trabajo o la escuela y viceversa (Metró-

3 Televidencia es un concepto desarrollado por Orozco (1996) para comprender lo que pasa más allá del momento de la exposición televisiva. En tal sentido, por televidencia entendemos el antes, durante y después de la exposición televisiva. 
poli, 2025, 2005). Es en este contexto que, para mi sorpresa, la televisión se vuelve un acompañante de los automovilistas. Y no sólo de los pasajeros que se ubican en los asientos traseros, también de los conductores. Claro, esto si no se ve no se cree. Pero es así. Los atascos en el tráfico son tan frecuentes y se prolongan por tanto tiempo, que es posible que el propio conductor del automóvil consuma los más variados programas de televisión. Mientras hoy las camionetas vienen equipadas con pantallas reproductoras de DVD, pensadas para emitir películas, sobre todo para niños, los automóviles que circulan por la ciudad de México instalan en sus tableros pequeños receptores de televisión, también pensados para otros contextos de consumo, seguramente la cocina de la casa. Estos aparatos, alimentados con batería, captan la señal de televisión abierta a través de las antenas de conejo. A las películas, entonces, podemos sumar los programas de espectáculos o de noticias que coinciden con la vuelta del trabajo, momento que se convierte, según hemos podido observar, en habitual. Aunque el tipo de consumo de la televisión que se realiza en los automóviles no es el tema central de este trabajo, queríamos dejar apuntada esta línea de acción para futuras investigaciones, que complementarán la que aquí presentamos. ${ }^{4}$

\section{MÉTODO}

Es importante destacar que para este trabajo, tanto en su etapa cuantitativa como en la cualitativa, se contó con un equipo de investigadores de campo que coadyuvó en la producción de la información empírica. ${ }^{5}$ Di-

4 Será relevante contrastar los resultados de una investigación sistemática sobre el consumo de televisión en los automóviles en la ciudad de México con lo que sucede en otras megalópolis. Esto lo señalo, ya que la investigadora brasileña Maria Immacolata Vassallo de Lopes (2009) apuntó en el pasado congreso de la International Association of Media and Communication Research (IAMCR), que una de las razones por la cuales se había constatado una baja en el consumo de televisión en Brasil responde a que los habitantes de grandes ciudades como Sãu Paulo se demoran en el tráfico por largo tiempo, impidiendo el consumo televisivo.

5 Agradecemos la participación en el trabajo de campo de los estudiantes de 
cho esto, presentamos aquí los primeros resultados de una investigación que se desarrolló en dos etapas. La primera implicó un trabajo sobre los mercados cuantitativo, indagando, específicamente: número de locales de venta de productos; número de locales de expendio y consumo de alimentos; número de aparatos de televisión (total); número de aparatos de televisión de cara al público; número de aparatos de televisión de espaldas al público; número de aparatos de televisión encendidos (canal y programa); número de comerciantes mirando televisión; número de consumidores mirando televisión; número de cocineros y meseros mirando televisión, $\mathrm{y}$ finalmente, números de comensales mirando televisión.

Los siguientes condicionantes deben tomarse en cuenta para la lectura de los resultados que se presentarán a continuación: día y horario de la visita a los mercados por parte de los evaluadores 6 y, aunque debería considerarse en primer lugar, el período de tiempo que permanecen abiertos los mercados: de 9:00 a 18:00 hrs. en promedio. La información se generó entre el 4 y el 21 de junio de 2008.

Según los datos del primer informe de gobierno 2006-2007 del Gobierno del Distrito Federal (2008, p. 48) 317 mercados públicos ${ }^{7}$ se distribuyen en las 16 delegaciones que comprende la ciudad de México: 15 en Álvaro Obregón; 19 en Azcapotzalco; 16 en Benito Juárez; 20 en Coyoacán; 5 en Cuajimalpa; 37 en Cuauhtémoc; 50 en Gustavo A. Madero; 16 en Iztacalco; 20 en Iztapalapa; 5 en Magdalena Contreras; 17 en Miguel Hidalgo; 9 en Milpa Alta; 18 en Tláhuac; 17 en Tlalpan; 40 en Venustiano Carranza y 11 en Xochimilco. De éstos, determinamos una cuota de $10 \%$ para realizar el relevamiento cuantitativo, lo que resulta un total de 35 mercados.

la licenciatura en Comunicación y Cultura de la Universidad Autónoma de la Ciudad de México, plantel San Lorenzo Tezonco: María del Carmen Mendoza, Nancy Malacara, Isabel Argüello, Anna Ahedo y Edgar Guzmán.

6 Las visitas se distribuyeron de la siguiente manera: 21 de lunes a viernes: 10 matutinas y 11 vespertinas; 12 durante el fin de semana: 4 matutinas y 8 vespertinas.

7 En esta investigación consideramos sólo los mercados públicos establecidos y reconocidos por el Gobierno del Distrito Federal, descartando los mercados ambulantes (tianguis). 
En la segunda etapa, ahora de tipo cualitativo, se realizaron 12 observaciones, de carácter participante, en distintos mercados de la ciudad de México, siguiendo tres tópicos: espacios, sujetos y prácticas sociales (con particular acento en los usos sociales de la televisión). Para ello, y en función de los resultados cuantitativos, se decidió tomar como espacio específico de observación dentro de los mercados a los locales de expendio y consumo de alimentos, ya que en éstos se observó la mayor cantidad de sujetos interactuando con la televisión durante la primera etapa de la investigación. Para cada observación se dedicó entre 2 y 4 horas (de 13:00 a 17:00 hrs.) período de tiempo en que se concentra el consumo de alimentos y que, por tanto, entendemos necesario para aprehender las características del espacio y tener un panorama suficiente de los sujetos, sus flujos y sus prácticas. La elección de los mercados fue aleatoria entre los 35 mercados en que se desarrolló la observación cuantitativa, tratándose de un estudio exploratorio a partir del cual diseñar nuevas estrategias de investigación para la comprensión de la interacción entre las audiencias y la televisión en el espacio público.

\section{RESULTADOS DE LA OBSERVACIÓN DEL CONSUMO TELEVISIVO EN LOS MERCADOS PÚBLICOS DE LA CIUDAD DE MÉXICO}

Entre los 35 mercados comprenden un total de 9,289 locales comerciales. De éstos, 6,251 son puestos de venta de productos y 3,038 locales de expendio y consumo de alimentos. En total, se contabilizó la existencia de 1,027 televisores, lo que significa, en promedio, que $11 \%$ de locales cuenta con un aparato. De éstos, 753 televisores están dispuestos de cara al público, mientras que 274 lo están de espaldas al mismo, es decir, dispuestos para el consumo particular, único y privado (/ "público") de los locatarios. En el momento de la visita a los mercados, 767 televisores se encontraban encendidos y 251 apagados. Esto último significa que el porcentaje de encendido era de $75 \%$.

Ahora bien, ¿qué canales estaban sintonizados en el momento de la visita? Los resultados indican lo siguiente: con mucha diferencia por sobre los demás se destacan los canales 7 y 13 de TV Azteca, y 2 y 5 de Televisa. Éstos cuentan con 163 (21\%) y 157 (20\%); 150 (19.5\%) y $119(15.5 \%)$ televisores sintonizados respectivamente. Es significativo 
que $76.7 \%$ de la cuota de pantalla se reduzca a cuatro canales de dos televisoras. Si nos quedáramos con este grupo de canales, TV Azteca y Televisa estarían en una posición equitativa de cuota de mercado. Sin embargo, si sumamos al segundo grupo de canales sintonizados, Televisa supera por un buen porcentaje a TV Azteca. En este segundo grupo se encuentran dos canales de la corporación Televisa: canal 9 (Galavisión) y 4, quienes tienen una cuota de encendido de 89 y 40 aparatos de televisión respectivamente. Estos dos canales, en conjunto, suponen $16.8 \%$ de los aparatos encendidos, lo cual, sumado a $19.5 \%$ de canal 2 y $15.5 \%$ de canal 5 , resulta un total de $51.8 \%$. Es decir, entre las dos empresas de televisión privada más importantes de México, ambas de sistema abierto, suman un $93.6 \%$ de la cuota de pantalla sintonizada en los mercados públicos de la ciudad de México. El restante $6.4 \%$ se lo reparten los canales $11,22,28,34,40,53+$ TV y EXA TV, que en conjunto sumaron un total de 33 aparatos sintonizados en su frecuencia; los canales de paga MTV, Tele Hit, Ritmoson, TNT, ESPN, Fox Sports, con un total de 20 aparatos sintonizados; 13 aparatos con emisión de contenidos de DVD, y finalmente, un total de 13 aparatos con una señal que los investigadores de campo no pudieron identificar (1.5\%), lo cual constituye el margen de error de un estudio en este rubro.

Otro dato importante arrojado por la investigación tiene que ver con los géneros y programas más consumidos. Estos son: las transmisiones de fútbol con un total de 214 (28\%); las películas con 96 (12.5\%); las telenovelas con un total de 96 (12.5\%); las noticias con $70(9 \%)$; los dibujos animados, caricaturas y anime con 46 (5.9\%). Un segundo grupo integrado por los programas de variedades y espectáculos, con la especificación de "Hoy", "Venga la alegría", "Tempranito", "De poca" y "Se Vale" reúnen 84 emisiones, es decir, 10.9\%. Al mismo tiempo, si a las 214 transmisiones de fútbol sumamos las 38 emisiones de programas deportivos, entre las cuales destacan las emisiones de lucha libre, significan $32.8 \%$ del total. El último género con cierto peso específico es el de los programas musicales, que cuenta con 16 emisiones (2\%). Finalmente, un conjunto de programas varios suman 85 emisiones, pero ninguno de ellos resulta significativo por sí mismo.

Con lo anterior, podemos constatar un marcado desequilibrio: primero entre los deportes, en particular el fútbol, y cualquier otro género, 
y segundo, entre la ficción y la información. La ficción, en conjunto, se posiciona en segundo lugar con $25 \%$, mientras que los contenidos informativos representan $9 \%$ del total, superados por las emisiones de espectáculos y variedades con $10.9 \%$.

Por último, el estudio cuantitativo nos dice que de 2,984 personas que estaban mirando la televisión al momento de la visita de los investigadores 932 (31.2\%) son comerciantes; 651 (21.8\%) consumidores-clientes; $338(11.3 \%)$ cocineros y meseros de los locales de expendio y consumo de alimentos, y 1,063 (35.6\%) son comensales-clientes.

\section{USOS SOCIALES DE LA TELEVISIÓN \\ EN LOS MERCADOS PÚBLICOS DE LA CIUDAD DE MÉXICO}

En todos los mercados la Virgen de Guadalupe ocupa un lugar privilegiado, siempre iluminada por veladoras y adornadas con el mayor esmero y creatividad. La estructura de los mercados también es bastante similar de uno a otro. Suelen tener múltiples entradas y salidas, coincidentes con algunos de los pasillos que organizan la distribución de los puestos. Estos pasillos pueden ser de distinto ancho y largo. Todos están conectados de alguna manera. Los mercados, según su dimensión, pueden resultar laberintos para los visitantes esporádicos, mientras que para los visitantes asiduos es un espacio perfectamente establecido, certero en la estructuración de sus espacios y sus flujos. Si bien suelen tener una organización por rubro, ésta no es una regla. Los puestos de expendio y consumo de alimentos pueden estar reunidos en un área específica, pero también es muy frecuente encontrarlos desperdigados a lo largo y ancho del mercado. Incluso, estas dos lógicas pueden compartirse en un mismo mercado.

Entre los rubros de venta de productos encontramos los puestos de abarrotes, carnicerías, tiendas de ropa, materias primas, florerías, tlapalerías, reparaciones varias, pollerías, mercerías, paleterías, cremerías, productos para el hogar, fruterías y verdulerías, tortillerías, venta de hierbas, granos, dulces, celulares, zapatos, productos para pájaros, productos para toda ocasión y un largo etcétera.

Además, el mercado tiene espacios destinados a la carga y descarga de productos, basurero, sanitarios y la administración del mismo. 
Podemos señalar los siguientes tipos de sujetos-actores de las prácticas sociales que se desarrollan en los mercados: a) los comerciantes (según la zona de la ciudad pueden ser vecinos del mercado o trabajadores golondrinas); 8 b) cocineros, meseras y garroteros; c) los vecinos de la colonia en que se emplaza el mercado, quienes compran materias primas, contactan operarios para realizar obras en sus casas o, muy frecuentemente, consumen alimentos en el propio mercado; d) los trabajadores golondrina que laboran en las inmediaciones del mercado y que asisten, fundamentalmente, a comer al mercado en los descansos intermedios de la jornada laboral; e) los vendedores de piratería, que no tienen puestos asignados y se ubican en la periferia del mercado, especialmente a los lados de las entradas y salidas; f) considerando una periferia más extensa, las calles aledañas suelen ocuparse por puestos semiestablecidos, sobre las aceras o las mismas calles. Claro, éstos nunca llegan a cerrar las vialidades como ocurre con los tianguis (mercados ambulantes) ${ }^{9}$ cuando se emplazan en tal o cual calle.

\section{USOS SOCIALES DE LA TELEVISIÓN EN TORNO A LAS COCINAS}

Entre los comercios de expendio y consumo de alimentos, encontramos puestos de tacos, tortas, jugos y cócteles, cocinas económicas ${ }^{10}$ (también

8 Por trabajador golondrina entiendo a aquellos que no viven en la zona en que trabajan y deben desplazarce cada día de una zona habitacional a su lugar de trabajo. El concepto se retoma de aquellos migrantes que se desplazan temporalmente a los lugares de trabajo y, una vez concluido retornan a sus lugares de origen.

9 Los mercados objetos de este estudio se consideran mercados públicos fijos, diferentes a los mercados ambulantes (tianguis) y a las tiendas de autoservicio o supermercados. Las tres se proponen como centros de abasto de los habitantes de la ciudad.

10 "Dentro de este rubro de cocinas económicas encontramos locales que expenden alimentos preparados y que generalmente son consumidos dentro del mismo local como son: locales que preparan antojitos mexicanos, que preparan jugos y postres de frutas, torterías, taquerías y locales que preparan comidas corridas" (Luna, Pérez, Silva, Ochoa \& Barron, 2000, p. 25). Es necesario 
suele denominárselas como cocinas de "comida corrida"11 o "comida preparada"), sopes y panes (cocoles). Las cocinas económicas, según el tamaño y la ubicación, disponen taburetes formando una sola línea a un lado de la barra que la separa del pasillo. Allí sirven la comida a los comensales, quienes frecuentemente son atendidos por el propio cocinero o cocinera. Otras, con mayor espacio, disponen mesas de plástico o madera (generalmente cedidas por alguna marca de refrescos o cervezas), cuadradas o rectangulares, pintadas de vivos colores. En estas últimas, la presencia de meseras y garroteros ${ }^{12}$ se vuelve imprescindible. En todos estos puestos la comida se elabora a la vista del consumidor. También, encontramos uno o más televisores suspendidos de las paredes a una altura suficiente como para que el comensal pueda visualizarlo, lo que dificulta la interacción con la televisión es el espacio del que dispone la cocina. En las que sólo tienen una barra, el televisor se ubica a corta distancia, facilitando la recepción. En cambio, en las cocinas con un espacio de mesas y sillas, según la disposición de los lugares, el consumidor puede quedar cerca o lejos del televisor, lo cual afecta la posibilidad de oír lo que se emite y a veces, incluso, dificulta la visión. A propósito de esto, nos parece significativo señalar que, en estas circunstancias, hay productos comunicativos que resisten mejor las condiciones de recepción, el contexto inmediato. En este sentido, las transmisiones deportivas no requieren necesariamente del audio; pueden ser seguidas sin mayor dificultad, sobre todo desde que las transmisiones incorporan textos informativos acerca del resultado y el tiempo en que se desarrolla la prueba o el partido de fútbol. Por otro lado, hay productos que no requieren de una visión definida y nítida, por ejemplo aquellos productos de los canales de mú-

precisar que por "torta" debe entenderse "bocadillo o sandwich". En tanto que cóctel, en el contexto de los mercados, es una "ensalada de frutas picadas, ocasionalmente bañada con miel o yogurth".

11 La comida corrida es un menú que se ofrece en fondas y pequeños restaurantes, que suele ser muy económico, donde comen empleados, estudiantes y otros sujetos de sectores populares. El menú consiste en sopa, guisado y un postre y la duración del servicio, a lo cual responde el nombre, es de aproximadamente 30 minutos.

12 El garrotero es quien limpia y recoge las mesas tras el servicio. 
sica. Aunque el video que acompaña la música pueda resultar por demás interesante, la sustancia de lo que se transmite es el sonido. El vapor y el vaho de las cocinas también pueden interferir en la recepción televisiva. Muchas veces los televisores, por lo limitado del espacio, pueden estar ubicados justo sobre las ollas. Un tercer elemento de interferencia, otra vez sobre el audio de la emisión, puede resultar la cercanía o lejanía a la calle, el tráfico que por ella se produce, las horas de mayor afluencia de consumidores en el mercado, cuya consecuencia, además de los apretujones, es que los decibelios van en ascenso en todas las conversaciones. Este efecto no sólo remite a las personas, sino también a los televisores que afloran en los diversos puestos, de comida o de productos. Así lo expresan algunos cocineros:

Pues ya estamos acostumbrados a ver la televisión con el ruido de los carros (Guadalupe, mesera).

No mucho. Te acostumbras al ruido (Carlos, mesero).

Una característica particular de estas interacciones la encontramos en la misma definición de las cocinas: corridas o rápida. Se come rápidamente en una pausa de la jornada laboral, y rara vez este tiempo coincide con el inicio y el final de un producto televisivo. A la vez, quienes trabajan en las cocinas no pueden prestar una constante atención a la televisión. Por esta razón, generalmente, el consumo televisivo es fragmentario. Esta segmentación no sólo se da porque los tiempos de los comensales, meseros y cocineros no coincidan con el inicio y fin de un programa, sino también porque no siempre estos sujetos mantienen una atención constante sobre la televisión. Muchas veces se ojea el monitor, otras sólo se lo ignora y otras tantas se lo tiene como ruido de fondo, como paisaje sonoro y visual:

La tenemos prendida y pues la estamos escuchando. A veces sí volteamos cuando pasamos (Guadalupe, mesera).

A veces sí. Si no la veo, la oigo (Ignacio, vendedor).

Más que nada la escucho (Alex, comensal). 
Esta práctica no sólo nos habla de usos y consumo. Al mismo tiempo nos abre una pista para explorar el lenguaje audiovisual de la televisión mexicana de carácter abierto que es la que consumen según los datos referidos en la primera parte de este trabajo donde la imagen parece no ser imprescindible para seguir un programa.

Por otro lado, los consumidores suelen espiar lo que está viendo el vendedor mientras éste lo despacha (lo cual no es despreciable si consideramos los resultados del consumo presentados más arriba, que nos dicen que 651 (21.8\%) de 2,984 personas que estaban viendo televisión eran consumidores-clientes). Puede quedarse mirando más allá del tiempo que dura la compra-venta si el producto lo atrae sobremanera. Esto último suele pasar con mayor frecuencia cuando se trata de un partido de fútbol, pero no es lo habitual. Los compradores suelen convertirse en flaneurs ${ }^{13}$ televisivos:

Pues yo digo que no, sólo que haya fútbol porque la prende mi esposo y se quedan algunas personas viendo (Rosa María, vendedora).

Hay unos que se quedan, hay unos que se van, depende (David, vendedor).

Los comensales pueden ser televidentes más estables, aunque su consumo sea fragmentado. En este sentido, de la observación se desprende que los comensales suelen permanecer en el lugar alrededor de 30 minutos.

Para meseras, meseros, cocineras o cocineros su consumo televisivo marca el tiempo de descanso o el final de la jornada laboral:

Cuando no hay gente si me siento y veo la tele. (Guadalupe, mesera).

Cuando no hay gente o estamos comiendo (Marta, cocinera).

Sólo después de las 6:00 de la tarde, ya que se termina el trabajo (Rosa María, vendedora).

13 Flaneurs es un viajero. En este caso, lo comprendemos como un sujeto que ve la televisión mientras está de paso por el mercado. 
Cuando no hay clientes (Ignacio, vendedor).

Para los vendedores de otro tipo de productos, en cambio, se reitera que la televisión es una compañía y una distracción a lo largo de todo el día, no sólo del momento de descanso y finalización de labores. "Simplemente la vemos", señala uno de los vendedores describiendo el consumo de televisión como una práctica naturalizada, parte de las costumbres cotidianas. Se enciende y apaga como se abre o cierra el puesto. En este sentido, el uso de la televisión en los mercados no sólo señala e instaura el tiempo de espera del servicio, sino que encuadra el tiempo y el espacio laboral en los mercados.

También interviene en los usos sociales de la televisión el que se asista al mercado a comer sólo o acompañado. Acudir con la familia, los amigos, la pareja o los compañeros de trabajo puede afectar la interacción. A veces, como en el caso de los partidos de fútbol, la televisión se impone sobre las relaciones interpersonales. En contraste, otros productos no logran imponerse con tal nitidez sobre lo interpersonal; es más, la mayor parte de las veces, lo interpersonal se impone sobre las interacciones mediáticas:

Acompañado, no pues casi no le pongo atención a la televisión, pues estoy más platicando con la que venga conmigo, cuando vengo solo, le presto mas atención (Rubén, comensal).

En algunas ocasiones, yo creo que sí, cuando vengo solo y no tengo con quien platicar (Alex, comensal).

Sí, cuando vienen solitos están mirando más la televisión (Daniel, hijoayudante de cocinero).

Interrogando a cocineros, meseros y comensales sobre si la elección del lugar donde sentarse a comer depende de la ubicación del televisor, y aunque no siempre sucede, señalan que:

Sí. La televisión es más relevante que la música, sobre todo si hay partido o algún programa especial (Isabel, mesera). 
Regularmente sí, para tener una mejor perspectiva (Alex, comensal).

Es conveniente señalar que, como se puede constatar en el estudio cuantitativo, la televisión no siempre está prendida. En varias de las observaciones tuvimos que esperar a que la encendieran (voluntariamente) o cambiar de sitio. Esto, más que una dificultad, nos resulta un dato importante sobre el consumo televisivo, tanto como cuando la televisión está encendida. Es evidente que, para nuestro trabajo, cuando el aparato televisivo está encendido y hay público, el consumo se diversifica y la observación se enriquece. Pero no queremos dejar de subrayar, aunque parezca una obviedad, que mantener la televisión apagada es también un tipo de interacción. Por las razones que fueran y ya sea por una decisión racional o inconsciente, al igual que sucede cuando la prendemos: a veces buscamos prenderla y otras veces lo hacemos por rutina, casi como un gesto automático. De todas maneras, como pudimos constatar en algunas observaciones, cuando la televisión se prendió el lugar ganó en clientes. En tal sentido, podemos concebir a la televisión como un reclamo, una forma de atraer clientes, no sólo entretenerlos o acompañarlos durante la comida. Así lo afirman las cocineras y meseras:

Un lugar destinado a la venta de comida no es nada sin un televisor, tú sabes... atrae a la clientela y cuando ya está aquí la mantiene entretenida y hace que se olviden de sus problemas. Hay mujeres que me tienen tanta confianza que me cuentan su vida, $y$, a veces, sólo a veces, les doy uno que otro consejito (María, cocinera).

Pues sí, porque la televisión atrae (Isabel, mesera).

Porque mi patrona me dice para que entren más clientes. De hecho yo no prendo la tele, pero cuando ella sube me dice que por qué no prendo la tele (María Elena, mesera).

\section{FÚTBOL, FOCO DE ATENCIÓN}

Inicia la Eurocopa y el señor que atiende la pollería cambia del canal 13 al 7. Un cliente pregunta “¿quién juega?”, "España-Italia”, le respon- 
den. Otro cliente mira atento el televisor, su esposa termina de comprar y lo llama. Él le responde: "ya empezó, viste", comienzan a caminar pero el señor se inclina por última vez para ver el partido. Un tercer cliente, que pide un cuarto de chicharrón, ve el partido. Una vez que lo despachan permanece en el mismo lugar para seguir viendo el partido. Al rato pregunta al cocinero: "¿en qué canales va a salir?", "En el 5 y en el 7", le responde. El señor se despide y se va rápido. Uno de los meseros, que no tiene mucho trabajo, voltea hacia la televisión y se recarga en el refrigerador donde está la carne para ver mejor el aparato. “¿Qué va a llevar?", pregunta el cocinero a un nuevo cliente. El señor pide un taco y se sienta. No deja de mirar el televisor. Otro cliente pide ocho tacos para llevar y en pequeños ratos mira para la televisión. En tanto, un cliente que esperaba su orden se pone de pie y, mientras le cobran, sigue viendo el partido. En eso llega un muchacho que empieza a comer mientras ve el partido. Termina el primer tiempo y el joven se marcha. Un nuevo cliente llega en el entretiempo y pregunta si están viendo el partido, y agrega: “¡ojalá que gane España!”. El cocinero responde que "se van a ir a ceros y no creo que gane España. ¿Qué?, ¿tiene tendencia gachupina?". El cliente contesta: "no, sólo es para hacerlo enojar". Ríen y se despiden. El carnicero vecino vuelve a salir y de nuevo se recarga en la pared para ver el partido.

Podríamos seguir el relato de las idas y venidas en torno a la trasmisión de fútbol. Lo que queda claro, y tiene su reflejo en los datos cuantitativos que señalan al fútbol como el programa más sintonizado en los mercados de la ciudad de México, es que el fútbol es uno de los programas que más atención genera en los comerciantes y clientes. Esta afirmación, por supuesto, corresponde al período y los lugares en los que se desarrolló el trabajo de campo de la investigación. Debería explorarse en distintos períodos el consumo televisivo en los mercados públicos para constatar si se trata de una constante o se observan distintos comportamientos.

No obstante, preguntando sobre si alguna vez han pedido prender la televisión o sintonizar un canal en particular, nos encontramos con que los hombres, recurrentemente, solicitan sintonizar encuentros de fútbol:

Cuando hay fútbol pos sí, cuando no, lo que sea (Rubén, comensal). 
Los partidos en fines de semana (Sandra, mesera).

Pos si hay algún partido (Alex, comensal).

No. Bueno, hay clientes que sí piden que se les ponga el fútbol (Carlos, cocinero).

Si a estas solicitudes, que como decíamos configuran una práctica recurrente, le sumamos los datos del consumo televisivo registrado en la primera parte de este trabajo, el fútbol demuestra un contenido programático destacado en los mercados públicos, lo cual ya es habitual en otro tipo de espacios, como cantinas y bares, donde éste y los deportes en general son parte de la oferta primaria de consumo, lo cual difiere respecto de la oferta primaria de los mercados: productos y servicios alimenticios.

Esta diferencia, que parece menor, se vuelve relevante si la comparamos con los estudios de Grimson, et al. (1999), para el caso argentino, y Gastaldo et al. (2006), para el caso brasileño, donde la recepción colectiva en los bares es intencional, buscada, mientras en los mercados de la ciudad de México el fútbol, más que un fenómeno colectivo es algo inevitable. No se busca ver el partido en grupo en los mercados, sino que se ve fútbol en el mercado porque tocó estar ahí en el momento del partido, ya sea porque se está trabajando en él o porque la mujer lo exhortó a acompañarla a realizar las compras. A diferencia de un chaparrón inesperado, del cual intentamos huir o cubrirnos, la sorpresa de encontrarnos con el partido ahí es un reclamo. Los hombres se paran frente al televisor como si se pararan a disfrutar del chaparrón. No importa qué estaban haciendo o qué venían a hacer. El fútbol, en ese momento, lo es todo. En lo que sí se coincide con los estudios mencionados anteriormente, es que el fútbol y sus transmisiones televisadas en particular, son un fenómeno casi exclusivamente masculino. Aunque se presentan casos aislados de mujeres que gustan del fútbol tanto como los hombres, en las observaciones esto no se manifestó. Queremos destacar que dicha interacción es atípica para los mercados, ya que su público, a diferencia de bares y cantinas, no tiene una predominancia masculina. Más bien al contrario, el mercado tiene 
la particularidad de reunir tanto a hombres como mujeres en un mismo espacio. Como otra línea de acción, entonces, dejamos apuntada la interrogante sobre la recepción colectiva de partidos de fútbol en bares y cantinas de la ciudad de México.

\section{APUNTES FINALES A MANERA DE CIERRE}

Aunque los usos sociales de la televisión en el espacio público de la ciudad de México están totalmente naturalizados, su investigación recién comienza. Este trabajo hace foco en un espacio público determinado: los mercados públicos de la ciudad de México, y registra un abanico de usos sociales de la televisión, así como su consumo en $10 \%$ de los mercados de la ciudad.

El mercado es un espacio de compra-venta de productos y servicios. Pero además, la irrupción de la televisión desde los puestos y en las cocinas establece otro tipo de intercambio, un consumo cultural que rebasa la relación mercantil. Este consumo cultural constituye un consumo de segundo orden, cuando la compra-venta de productos y servicios y la alimentación constituyen la actividad prioritaria, origen de la necesidad que conduce a los consumidores y comensales a los mercados. Los mercados no son espacios pensados para ver televisión, pero se ve. Así, aunque concebidos originalmente como centros de abastos de productos alimenticios para los habitantes de la ciudad de México, ${ }^{14}$ en ellos se desarrolla este consumo secundario, desapercibido en primera instancia, que es un consumo cultural, particularmente de la televisión, reconfigurando lo que allí sucede.

En este artículo no exploramos la apropiación de los contenidos, lo cual apuntamos como una siguiente etapa en la investigación de los

14 "En un diagnóstico elaborado por la Secretaría de Desarrollo Económico (SEDECO), los mercados, concebidos como los pilares del sistema de abasto alimenticio para la Ciudad hace cinco décadas y media, compiten en condiciones de desventaja con las tiendas de autoservicio en el aspecto técnico, normativo y operativo. Según fuentes de la SEDECO, el 52\% de la demanda de consumo la cubren los autoservicios y el $20 \%$ los mercados. Este porcentaje contrasta con el 60\% que abastecían los mercados en un principio" (Osorio, 2007, p. 1). 
usos de la televisión en los espacios públicos; lo que sí indagamos es el consumo y los usos sociales descritos más arriba.

Queremos subrayar aquí lo siguiente: casi 3,000 personas estaban viendo la televisión al momento de observar el consumo. De estos, $31 \%$ fueron comerciantes; $22 \%$ consumidores-clientes; $11 \%$ cocineros y meseros de los locales de expendio y consumo de alimentos, y $36 \%$ comensales-clientes.

También es relevante señalar que en los 9,289 locales se encontraron un total de 1,027 televisores (11\% de locales tienen televisión), con un encendido de 75\%, concentrando Televisa, y TV Azteca 94\% de cuota de pantalla.

Por último, destacar que se encontró un marcado desequilibrio entre los géneros programáticos sintonizados: 28\% fútbol (33\% deportes en conjunto), $25 \%$ ficción y $9 \%$ información. Como señalábamos más arriba, este desequilibrio puede adjudicarse al período en que se desarrolló el trabajo de campo de la investigación y deberá confirmarse replicando la investigación en distintos períodos del año.

En cuanto a los usos sociales, como hemos visto, van desde la absoluta ignorancia de la televisión hasta la total concentración. Se la consume en grupo o solo, casi siempre de forma fragmentaria, que es la característica más significativa de los usos sociales de la televisión en este contexto específico.

En el marco de los usos sociales, los resultados de nuestra observación coinciden con los obtenidos por Rodríguez (2009) respecto de los usos sociales en las áreas de comida de un centro comercial, los cuales responden a los usos estructurales ambientales (sonido de fondo, compañía y entretenimiento) y reguladores (organización del tiempo y la actividad, pautas de conversación) señalados por Lull (1997).

Otra característica de los usos sociales de la televisión en los mercados, como se pudo observar a lo largo del artículo, es que la presencia del televisor en las cocinas se constituye en una oferta secundaria que puede definir la decisión de los clientes para sentarse a comer en las cocinas. Complementariamente, cuando el espacio lo permite, la televisión se convierte en el vértice a partir del cual se disponen los comensales.

Si bien generalmente se puede considerar al consumo televisivo en los mercados como un consumo fragmentado, que no responde al inicio 
y final de un programa, también encontramos que hay momentos, de acuerdo a los clientes-comensales y los programas que se están emitiendo, que llevan a los clientes-comensales a permanecer en el lugar más allá de la satisfacción de la actividad primaria: comer, comprar. En este sentido, observamos que no designa sólo el tiempo de la espera, como lo señala puntualmente McCarthy (2001) ni regula exclusivamente el paso de una actividad a otra. Por momentos, el consumo televisivo en los mercados pasa de un segundo orden al primer plano, haciendo incluso que la persona (cliente-comensal) permanezca más tiempo en el lugar que el que requiere el servicio.

En tal sentido, como señalamos en la introducción del artículo, no sólo la ciudad de México resulta un contexto cultural distinto al descrito por Silverstone en Televisión y vida cotidiana (1996). El mercado constituye un contexto de interacción específico con la televisión. Quiero subrayar aquí la importancia de la mediación situacional (Orozco, 1996) en las prácticas de consumo televisivo. En otra parte, con el objetivo de analizar la producción y crisis de sentido de audiencias multiculturales en situación de interculturalidad (Repoll, 2009), ya exploré esta mediación, señalando que la familia no es la única unidad de análisis. En dicho trabajo la mediación situacional estaba dada por la situación de interculturalidad y la situación de no-familiaridad. En este caso, aunque suelen concurrir familias completas a los mercados, ya sea que vayan de compras, a comer o a ambas cosas, por lo general el consumo de televisión no responde a una lógica familiar, sino a un consumo individual o en grupos de amigos o trabajadores en el caso de los clientes-comensales y de forma individual o con los propios compañeros de trabajo en el caso de las y los vendedores, cocineros y meseros.

La mediación situacional que opera en los mercados no sólo se opone a la lógica del consumo familiar, donde la estructura de parentesco y la relación entre los géneros (hombre-mujer) asumen un papel cardinal en relación con el consumo televisivo y demás prácticas sociales, sino que, como hemos visto en la presentación de los resultados, configura un complejo haz de usos sociales de la televisión, estructurales (ambientales y reguladores) desde la perspectiva de Lull (1997).

Podemos abundar en las características de la mediación situacional en los mercados públicos: 
1. Dimensión espacial: a) espacio abierto con múltiples puestos dispuestos uno junto al otro y separados en bloques por los pasillos que comunican a las puertas que permanecen abiertas; b) en consecuencia de esto último, el movimiento de la calle que penetra con facilidad el espacio al interior del mercado; c) espacio laboral; d) espacio de consumo de alimentos y de productos materiales y simbólicos, y e) espacio de tránsito.

2. Dimensión temporal: a) el mercado supone para los trabajadores (vendedores, cocineros y meseros) el tiempo del trabajo, con sus respectivas pausas y momentos de descanso o espera de clientes; b) para los clientes-comensales implica un tiempo de consumo (de alimentos y productos materiales y simbólicos), como parte de las actividades domésticas o como pausa de la jornada laboral o escolar desarrollada en las inmediaciones de los mercados.

3. Dimensión relacional: a) se establecen relaciones comerciales entre clientes y comerciantes, y b) relaciones que exceden lo comercial, entre los mismos clientes o entre clientes y comerciantes. En esta segunda relación la televisión interviene en distintos niveles, según el grado en que se involucran los televidentes, ya sean vendedores, cocineros, meseros, clientes o comensales. Los grados de este involucramiento varían de acuerdo a distintas circunstancias, también situacionales: asistir solo o acompañado, interés por ver un determinado programa, busca de compañía o distracción, excusa para conversar, márgenes de decisión para intervenir en el encendido o en la sintonía de determinado canal o programa, disposición de tiempo para estar o para ver, etcétera.

4. Dimensión comercial: decisión de colocar uno o más televisores en el local de comidas como parte de la oferta de consumo, reconociendo que la comida (necesidad primaria que se busca satisfacer) no es suficiente para decidir dónde comer.

5. Dimensión comunicativa: en torno a los televisores dispuestos en las cocinas, se conforman audiencias de forma aleatoria y circunstancial que pueden constituirse en comunidades de apropiación de los contenidos emitidos o como agregado de sujetos audiencia. En todo caso, estas posibilidades responden a las relaciones que se establezcan y que describimos previamente. Por otro lado, siguiendo 
el concepto de televidencia, en dichos grupos (durante el momento de la exposición televisiva) convergen distintas experiencias subjetivas (el antes de la exposición, considerados como dispositivos estructuradotes de sentido) y, al mismo tiempo, esta particular exposición al medio se actualizará, muy posiblemente, en otro momento y, quizá, con otros sujetos (el después de la exposición televisiva).

Quedan muchas otras experiencias de consumo y usos sociales de la televisión en espacios públicos por explorar. En el texto apuntamos la interacción con la televisión en los automóviles particulares y la interacción en bares y cantinas. Podríamos señalar, como otra línea de acción específica, la interacción en el transporte colectivo, en particular la relación entre pasajeros y el canal Telebus. ${ }^{15}$

La utilización de otras técnicas de investigación podría, también, arrojar resultados importantes sobre la interacción con la televisión en los mercados. En particular, pensamos que la entrevista en profundidad nos puede ayudar a vislumbrar qué significa la televisión para los comerciantes y clientes, y al mismo tiempo, la encuesta nos permitiría establecer qué usos hacen y qué gratificaciones buscan estos mismos sujetos en la televisión.

Por otra parte, como ya señalamos, es imprescindible replicar el estudio en distintos períodos de tiempo a lo largo del año, para observar si se presentan variantes en el consumo televisivo, y también realizar una observación de los usos de la televisión a lo largo de la jornada, tratando de establecer diferencias entre actividades, sujetos y momentos del día.

Finalizamos señalando que la televisión no sólo forma parte del paisaje urbano, sino que es un elemento con el cual interactúan los ciu-

15 Telebus: es el servicio de información televisiva que se ofrece en los autobuses de la Red de Transporte de Pasajeros del Distrito Federal (RTP). Es necesario aclarar que este servicio no se ofrece en todas las unidades ni en todas las líneas que opera RTP. Por último, es pertinente señalar que la información se compone de imagen en movimiento y texto, careciendo de señal de audio. 
dadanos, en particular los sujetos trabajadores o estudiantes que por su ocupación no tienen tiempo para comer en casa, los clientes y los propios comerciantes de los mercados, y que, por tanto, forma parte constitutiva de su vida cotidiana, aun cuando no están en casa.

\section{Bibliografía}

CONEICC-Consejo Nacional para la Enseñanza y la Investigacion de las Ciencias de la Comunicación (2008). Catálogo CONEICC. Recuperado el 1 de agosto de 2008 de http://148.201.96.14/coneicc/

Fuentes, R. (1988). La investigación de comunicación en México. Sistematización documental 1956-1986. México: Ediciones de la Comunicación.

Fuentes, R. (2003). La investigación académica sobre comunicación en México. Sistematización documental 1995-2001. Guadalajara: ITESO.

Gastaldo, É., Leistner, R., Da Silva, R. \& McGinity, S. (2006). A bola no bar: apontamentos sobre a recepcao coletiva de jugos de futebol mediatizados em locais públicos. En N. Jacks, E. Reinhardt \& R. Sánchez (Orgs.), ¿O que sabemos sobre audiencias? Estudos latino-americanos (pp. 156-170). Porto Alegre: ALAIC/Arm@zém editorial.

Gobierno del Distrito Federal (2008). Primer informe de gobierno 2006-2007. Recuperado el 27 de mayo de 2008 de http://www.dgacd.df.gob.mx/

Grimson, A., Massota, C. \& Varela, M. (1999). Un electrodoméstico en la ciudad. Hacia una conceptualización del lugar de la televisión en el espacio público. En A. Grimson \& M. Varela, Audiencias, cultura y poder. Estudios sobre televisión (pp. 197-226). Buenos Aires: Eudeba.

Lozano, J. \& Frankenberg, L. (2008). Enfoques teóricos y estrategias metodológicas en la investigación empírica de audiencias televisivas en América Latina: 1992-2007. Comunicación y Sociedad, 10, 81-110. Nueva época.

Luna, J., Pérez, J., Silva, J., Ochoa, J. \& Barron, M. (2000). Diagnóstico de las condiciones sanitarias de los mercados públicos del Distrito Federal. Estudio de casos en el área de influencia del centro de salud Dra. Margarita Chorne y Salazar, Reporte de servicio social. México: UAM Xochimilco. 
Lull, J. (1997). Medios, comunicación, cultura. Aproximación global. Buenos Aires: Amorrortu.

McCarthy, A. (2001). Ambient television: visual culture and public space. Durham, North Carolina: Duke University Press.

Metrópoli 2025 (2008). Encuestas. Centro de Estudio para la Zona Metropolitana A. C. Recuperado el 9 de junio de 2008 de http://www. metropoli.org.mx/modules.php?name=Surveys

Morley, D. (1996). Televisión, audiencias y estudios culturales. Buenos Aires: Amorrortu.

Orozco, G. (1996). Televisión y audiencias. Un enfoque cualitativo. Madrid: Ediciones de la Torre.

Orozco, G. (1997). La investigación de la comunicación dentro y fuera de América Latina. Tendencias, perspectivas y desafíos del estudios de los medios. La Plata: Ediciones de Periodismo y Comunicación Universidad Nacional de La Plata.

Orozco, G. \& Padilla de la Torre, R. (2005). Los estudios de recepción en México. Un itinerario. En J. Lozano (Ed.), La comunicación en México: diagnósticos, balances y retos (pp. 147-166). Puebla: CONEICC/Tecnológico de Monterrey.

Orozco, G. \& González, D. (2009). Cuatro décadas de analizar la recepción de medios en México. En A. Vega (Coord.), La comunicación en México. Una agenda de investigación (pp. 69-89). México: UNAM/UJAT/UABC/AMIC.

Osorio, E. (2007, 17 de diciembre). Salen caros al DF los mercados públicos. Periódico Reforma. Sección Ciudad. México.

Repoll, J. (2008). Prácticas significantes. La ciudad desde la comunicación. En M. Hernández \& J. Alvarado (Coords.), De espacios urbanos. Una visión estética y social sobre la ciudad (pp. 38-51). México: CONACULTA/UNAM.

Repoll, J. (2009). Estudio de audiencias multiculturales. Tesis de doctorado. Barcelona: UAB.

Repoll, J. (2010). Arqueología de los estudios culturales de audiencia. México: UACM.

Rodríguez, S. (2009). El ritual televisivo. Las articulaciones que le dan sentido. Tesis de doctorado. México: UNAM. 
Silverstone, R. (1996). Televisión y vida cotidiana. Buenos Aires: Amorrortu.

Tufte, T. (1997). Televisión, modernidad y vida cotidiana. Un análisis de la obra de Roger Silverstone desde contextos culturales diferentes. Comunicación y Sociedad, 31, 65-96.

Vassallo de Lopez, M. I. (2009, 22 de julio). Obitel International Project. En el congreso de la International Association of Media and Communication Research (IAMCR). Ciudad e México. 